\title{
Supergiant fast X-ray transients versus classical supergiant high mass X-ray binaries: Does the difference lie in the companion wind?
}

\author{
P. Pradhan ${ }^{1,2}$, E. Bozzo ${ }^{3}$, and B. Paul ${ }^{4}$ \\ ${ }^{1}$ Pennsylvania State University, State College, PA 16801, USA \\ e-mail: pup69@psu.edu \\ ${ }^{2}$ St. Joseph's College, Darjeeling 734104, West Bengal, India \\ ${ }^{3}$ Department of Astronomy, University of Geneva, Chemin d'Ecogia 16, Versoix 1290, Switzerland \\ ${ }^{4}$ Raman Research Institute, Sadashivnagar, Bangalore 560080, India
}

Received 1 July 2017 / Accepted 27 November 2017

\begin{abstract}
We present a comparative study of stellar winds in classical supergiant high mass X-ray binaries ( $\mathrm{SgXBs}$ ) and supergiant fast Xray transients (SFXTs) based on the analysis of publicly available out-of-eclipse observations performed with Suzaku and XMMNewton. Our data set includes 55 observations of classical SgXBs and 21 observations of SFXTs. We found that classical SgXBs are characterized by a systematically higher absorption and luminosity compared to the SFXTs, confirming the results of previous works in the literature. Additionally, we show that the equivalent width of the fluorescence $\mathrm{K}_{\alpha}$ iron line in the classical $\mathrm{SgXBs}$ is significantly larger than that of the SFXTs (outside X-ray eclipses). Based on our current understanding of the physics of accretion in these systems, we conclude that the most likely explanation of these differences is ascribed to the presence of mechanisms inhibiting accretion most of the time in SFXTs, thereby leading to a much less efficient photoionization of the stellar wind compared to classical SgXBs. We do not find evidence for the previously reported anticorrelation between the equivalent width of the fluorescence iron line and the luminosity of SgXBs.
\end{abstract}

Key words. X-rays: binaries

\section{Introduction}

Supergiant X-ray binaries ( $\mathrm{SgXBs)}$ are usually divided into classical systems and supergiant fast X-ray transients (SFXTs). The SFXTs share many properties in common with classical systems (e.g., similar supergiant companions and orbital period distribution) and in all these sources high energy emission is mostly due to the accretion of stellar wind from the massive companion onto the compact object. Compared to classical systems, SFXTs show a much more pronounced variability, comprising sporadic short X-ray outbursts and fainter flares with fast rise times (tens of minutes) and typical durations of a few hours. Outside these events, the SFXTs have average X-ray luminosities that are 2-3 orders of magnitude lower than the classical systems with similar orbital periods (see, e.g., Walter et al. 2015, for a recent review). The presence of a neutron star (NS) as a compact object has been established by the detection of X-ray pulsations in classical $\mathrm{SgXBs}$ and in a few intermediate objects between SFXTs and classical SgXBs. There are no confirmed detections of pulsations for any of the known SFXTs ${ }^{1}$. Cyclotron lines, probing the strength of the NS magnetic field, have been

\footnotetext{
1 Different tentative spin period detections of the SFXT IGR J175442619 have been reported but never confirmed (Drave et al. 2012, 2014; Romano et al. 2015). Similar cases are those of the SFXT IGR J184830311 (Sguera et al. 2007; Ducci et al. 2013) and IGR J18410-0535 (Bamba et al. 2001; Bozzo et al. 2011).
}

detected in many classical systems, but only in one SFXT has some evidence been reported for a cyclotron feature at $\sim 17 \mathrm{keV}$ (Bhalerao et al. 2015). This was not confirmed by more recent observations (Bozzo et al. 2016).

The few models proposed to explain the extreme X-ray variability of the SFXTs are still a matter of debate. These include extremely clumpy stellar winds (in't Zand 2005), magnetic or centrifugal gates (Grebenev 2008; Bozzo et al. 2008), or the settling of a long-lasting quasi-spherical accretion regime (Shakura et al. 2012). In the latter two cases, it was shown that reasonably limited clumpy winds are needed to achieve the dynamic range of the SFXTs if their activities are sporadically boosted by the effect of the NS rotating magnetosphere or its interaction with the magnetized wind from the supergiant companion or both.

Both the magnetic or centrifugal gates and settling accretion regime are likely to inhibit accretion in SFXTs, explaining their subluminosity compared to classical SgXBs. Our current limited understanding of the SFXT phenomenology makes any comparative study between these sources and the classical $\mathrm{SgXBs}$ particularly interesting. In this paper, we exploit archival Suzaku (Mitsuda et al. 2007) and XMM-Newton (Jansen et al. 2001) observations to carry out a comparative analysis of the stellar wind properties in these two classes of systems. We focus on the measurement of the average absorbing column density associated with the stellar wind and the properties of the fluorescence iron line (centroid energy and equivalent width). 


\section{Observations and data reduction}

We only included $\mathrm{SgXBs}$ that are believed to be primarily windfed systems in our data set. Sources for which strong evidence was reported in the literature for the presence of an accretion disk were not included. A summary of all observations used for the present work is provided in Table 1 . Recent reviews by Walter et al. (2015) and Martínez-Núñez et al. (2017) provide an overview of the most relevant properties of each system. In contrast with other studies investigating the spectral variability on timescales comparable with the clumpy wind dynamics (see, e.g., Bozzo et al. 2011, 2016, 2017), in the present case we are interested in evaluating the wind properties on a larger scale. As clumps in the wind are known to give rise to variability over a hundred to thousand seconds (see, e.g., Walter \& Zurita Heras 2007, and references therein), their effect can be neglected when using integration times as long as several tens of kiloseconds. To quantitatively investigate the properties of the stellar winds in classical $\mathrm{SgXBs}$ and SFXTs, we thus extracted their average spectra from all publicly available Suzaku and XMM-Newton observations. We focused on deriving, from the fits to these spectra, a measurement of the absorption column density in excess of the Galactic value and fluorescence iron line properties. The first parameter provides an estimate of the average stellar wind density from which the compact object is accreting. The centroid energy and equivalent width (EW) of the fluorescence iron emission line are also key probes of the stellar wind properties. This feature originates from the fluorescence of the X-rays from the compact object onto the surrounding stellar wind and it is known that larger EWs correspond to denser winds (outside X-ray eclipses; see, e.g., Torrejón et al. 2010b).

We processed Suzaku data from one of the X-ray Imaging Spectrometer (XIS) units - XIS0 (0.2-12 keV; Koyama et al. 2007), using filtered cleaned event files obtained from the application of predetermined screening criteria ${ }^{2}$. For sources that showed jitters in the detector image, the event files were corrected via the aeattcorr and xiscoord tools to update the attitude information. For those sources affected by pileup, we discarded photons collected within the portion of the point spread function (PSF) where the estimated pileup fraction was $>4 \%$. This was carried out with the FTOOLS task pileest. The XIS0 spectra were extracted by choosing circular regions of $2^{\prime}$, $3^{\prime}$, or $4^{\prime}$ radius centered around the best-known source position, depending on whether the observation was made in $1 / 8,1 / 4$, or 0 window mode, respectively. Background spectra were extracted by selecting regions of the same size, as mentioned above, in a portion of the CCD that was not significantly contaminated by the source X-ray emission. Response files were created using the CALDB version "20150312".

XMM-Newton observation data files (ODFs) were processed using the standard Science Analysis System (SAS 14.0) and following the procedures given in the online analysis threads ${ }^{3}$. We primarily used data from the $\mathrm{PN}(0.5-12 \mathrm{keV})$ whenever available, as this instrument provides a better statistics compared to the Metal Oxide Semi-conductor (MOS) cameras (0.5-10 keV). The latter were used in all those cases in which the PN data were not collected or not usable. We did not make use of the Reflection Grating Spectrometers (RGS) data because of the limited band pass of this instrument and the need for the results to be

\footnotetext{
2 http://heasarc.gsfc.nasa.gov/docs/suzaku/analysis/ abc/

3 http://www. cosmos.esa.int/web/xmm-newton/ sas-threads
}

Table 1. Log of all used observations.

\begin{tabular}{|c|c|c|c|}
\hline Source & OBSID & & Effective exp. (ks) \\
\hline & $X M M-N e w t o n$ & Suzaku & \\
\hline & Classical SgXBs & & \\
\hline IGR J00370+6122 & 0501450101 & - & 16.2 \\
\hline $4 \mathrm{U} 0114+65$ & - & 406017010 & 106.6 \\
\hline Vela X-1 & 0406430201, 0111030101 & 403045010 & $118.9,53.3,104.7$ \\
\hline GX 301-2 & 0555200301, 0555200401 & $\begin{array}{l}403044020 \\
403044010\end{array}$ & $\begin{array}{l}58.1,46.0,61.8 \\
11.4\end{array}$ \\
\hline 4U 1538-522 & 0152780201 & 407068010 & $38.1,25.1$ \\
\hline IGR J16207-5129 & 0402920201 & 402065020 & $30.6,32.7$ \\
\hline IGR J16318-4848 & $\begin{array}{l}\text { 0154750401, 0201000201, 0201000301, } \\
\text { 0201000401, 0742270201 }\end{array}$ & $\begin{array}{l}401094010 \\
-\end{array}$ & $\begin{array}{l}23.3,17.8,21.0,97.3 \\
15.1,64.4\end{array}$ \\
\hline IGR J16320-4751 & $\begin{array}{l}\text { 0128531101, 0556140101, 0556140201, } \\
\text { 0556140301, 0556140401, 0556140501, } \\
\text { 0556140601, 0556140701, 0556140801, } \\
\text { 0556141001, 0201700301 }\end{array}$ & - & $\begin{array}{l}17.4,7.9,6.9 \\
6.1,9.8,1.9 \\
10.8,7.8,8.4 \\
6.5,44.5\end{array}$ \\
\hline IGR J16393-4643 & 0206380201,0604520201 & 404056010 & $8.5,6.3,50.5$ \\
\hline IGR J16418-4532 & 0405180501, 0679810201, & - & $22.7,11.1$ \\
\hline IGR J16493-4348 & - & 401054010 & 21.1 \\
\hline OAO 1657-415 & - & 406011010 & 84.7 \\
\hline 4U 1700-37 & $0083280101,0083280201,0083280301$ & 401058010 & $24.9,31.2,19.5,40.7$ \\
\hline EXO 1722-363 & $\begin{array}{l}\text { 0405640301, 0405640401, 0405640701, } \\
0405640801,0405640901\end{array}$ & $\overline{0}-06380401$ & $\begin{array}{l}4.2,5.6,19.2 \\
12.3,12.0,0.6\end{array}$ \\
\hline SAX J1802.7-2017 & $\begin{array}{l}\text { 0206380601, 0745060401, 0745060501, } \\
0745060601,0745060801\end{array}$ & - & $\begin{array}{l}9.5,37.3,14.3 \\
15.2,13.7\end{array}$ \\
\hline XTE J1855-206 & - & 409022010 & 42.2 \\
\hline 4U 1909+07 & - & 405073010 & 29.3 \\
\hline IGR J19140+0951 & 0761690301 & - & 34.1 \\
\hline & SFXTs & & \\
\hline$\overline{\text { IGR J11215-5952 }}$ & 0405181901 & - & 15.2 \\
\hline IGR J16195-4945 & - & 401056010 & 39.2 \\
\hline IGR J16328-4726 & $0728560201,0728560301,0654190201$ & - & $29.6,11.2,14.9$ \\
\hline IGR J16479-4514 & - & 406078010 & 51.8 \\
\hline IGR J17354-3255 & 0701230101, 0701230701 & - & $22.2,18.3$ \\
\hline IGR J17391-3021 & 0554720101,0561580101 & - & $34.0,24.3$ \\
\hline IGR J17544-2619 & $\begin{array}{l}0744600101,0679810401 \\
0154750601\end{array}$ & 402061010 & $\begin{array}{l}117.6,10.5,103.8 \\
2.5\end{array}$ \\
\hline SAX J1818.6-170 & 0693900101 & - & 21.5 \\
\hline IGR J18410-0535 & 0604820301 & 505090010 & $37.1,49.6$ \\
\hline IGR J18450-0435 & 0728370801,0306170401 & - & $14.9,15.2$ \\
\hline IGR J18462-0223 & 0651680301 & & 12.6 \\
\hline IGR J18483-0311 & 0694070101 & - & 36.9 \\
\hline
\end{tabular}

Notes. The effective exposure time for each observation is also indicated.

comparable with those obtained from the Suzaku data. All European Photon Imaging Camera (EPIC) spectra were corrected for pileup, whenever required. The correction was carried out using an annular extraction region whose inner radius was determined via the SAS tool EPAPLOT. Background spectra were extracted from a region located on the same CCD as that used for the target source, thereby avoiding any contamination from its emission. The difference in extraction areas between source and background was accounted for with the SAS BACKSCALE task. All spectra were rebinned in order to have at least 25 counts per energy bin and, at the same time, to prevent oversampling of the energy resolution by more than a factor of three. Individual Suzaku and XMM-Newton spectra were fit with a power-law model corrected for line-of-sight Galactic and local absorption with phabs and additional Gaussian components to take into account the presence of iron emission lines. The addition of a partial covering (pcfabs) or a thermal blackbody component was required in a few cases to account for the soft excesses in the $\mathrm{X}$-ray spectra. Since the data we used for the present work are not affected by low statistics issues, the detection of soft excess does not alter the measured values of the absorption column densities. We are thus confident about the representative nature of the obtained average values of the absorption column densities. Spectral fits were performed in all cases with XSPEC v12.9.0. For 

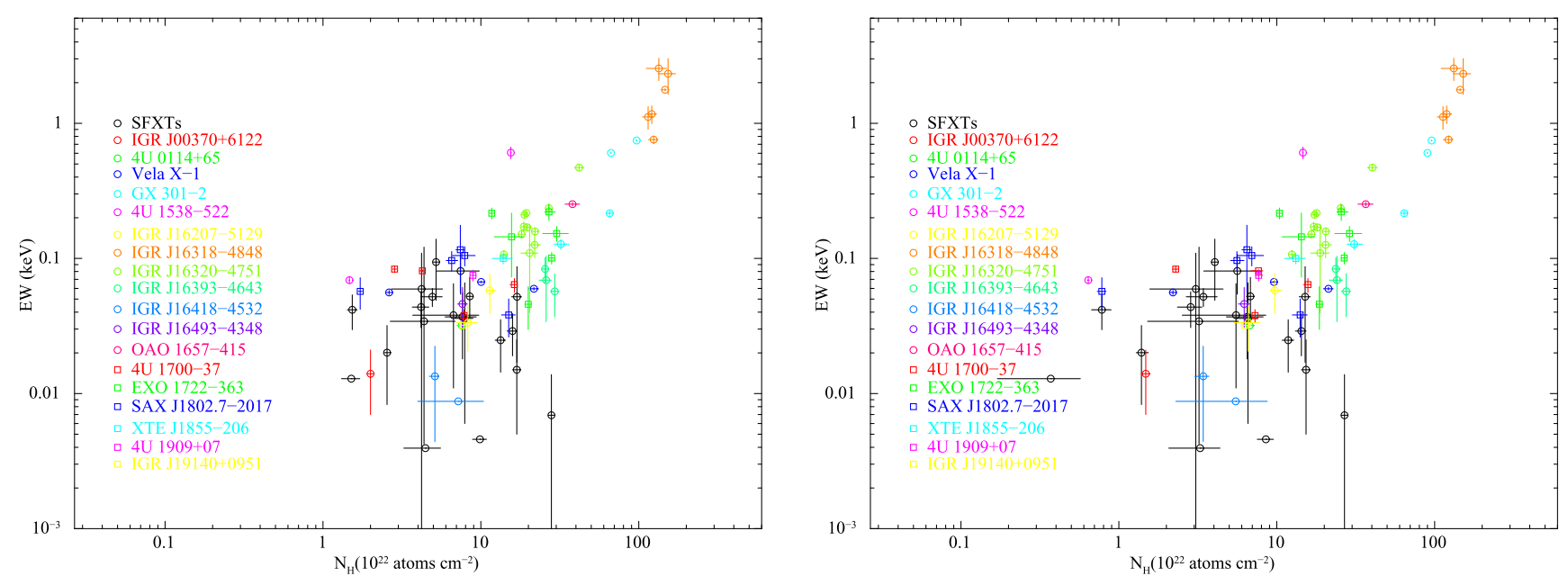

Fig. 1. Left: equivalent width vs. total column density of the $\mathrm{Fe}_{\alpha}$ line measured from all sources considered in this paper. The SFXTs are indicated in black, while a variety of colours have been used for classical SgXBs. Right: same as the left plot, but in this case the contribution of the Galactic absorption to the total $N_{\mathrm{H}}$ has been removed for all sources.

the eclipsing SgXBs, such as 4U 1700-37, Vela X-1, OAO 1657415, XTE J1855-026, EXO 1722-363, IGR J16195-4945, IGR J16479-4514, and 4U 1538-522, we did not use data collected during the X-ray eclipses. All spectra of the various sources are shown in Appendix A, together with the best-fit models and the residuals from the fits. We note that for the absorption models phabs and pcfabs, we used the default element abundances and cross sections in XSPEC (Anders \& Grevesse 1989; Verner et al. 1996) as the signal-to-noise ratio of the data at the lower energies does not allow us to discriminate between different possibilities.

\section{Results}

We plot the main findings of our analysis in Fig. 1, showing the measured values of the iron $\mathrm{K}_{\alpha}$ equivalent width $(\mathrm{EW})$ as a function of $N_{\mathrm{H}}$. For those sources in which a partial covering component was required, the value of the absorption column density reported in left panel of Fig. 1 includes all contributions ${ }^{4}$.

We also show in the right panel of Fig. 1 that the results do not change significantly if we remove for each source the expected Galactic contribution from the total absorption column density $^{5}$. The value of the Galactic absorption for all sources were estimated using the HEASARC online tool ${ }^{6}$.

We also show a plot of the iron $\mathrm{K}_{\alpha}$ line $\mathrm{EW}$ versus the X-ray luminosity in Fig. 2. The uncertainty on this last parameter is dominated for all sources by their poorly known distances. We

\footnotetext{
4 Total $N_{\mathrm{H}}=N_{\mathrm{H} 1}+N_{\mathrm{H} 2} * C_{\mathrm{V}}$, where $N_{\mathrm{H} 1}$ is the hydrogen column density along our line of sight to the source, $N_{\mathrm{H} 2}$ accounts for local absorption, and $C_{\mathrm{V}}$ is the covering fraction. The parameter $C_{\mathrm{V}}$ represents the fraction of the radiation from the NS that escapes from the variable and strong local absorptions. Large variations of this parameters have been recorded in different sources and ascribed to the presence of a largely variable and unstable accretion environment around the compact object (see, e.g., Malacaria et al. 2016). See also Table A.1.

5 We note that this test is carried out because if the measured value of Galactic absorption is several times larger than the online value, as is the case now, it is speculated to be due to the presence of a complex multicomponent absorber local to the source.

6 https://heasarc.gsfc.nasa.gov/cgi-bin/Tools/w3nh/ w3nh.pl
}

do not find any indication of the anticorrelation between the two parameters represented in this plot, which is at odds with the findings reported by Torrejón et al. (2010b) and Giménez-García et al. (2015). The red points on the top left side of the plot, which give the appearance of an anticorrelation, are from one source, IGR J16318-4848, in which the compact object is believed to be obscured for most of the time by a dense cocoon of material (Chaty \& Rahoui 2012).

\section{Discussion}

The results reported in the two panels of Fig. 1 show that there is a clear direct correlation between the absorption column density and the iron line EW in classical SgXBs and SFXTs. This is expected because the iron lines in these systems is produced by fluorescence of the X-rays from the compact object onto the surrounding stellar wind (George \& Fabian 1991), which is also the material giving rise to the measured local absorption column density (Inoue 1985). A larger $N_{\mathrm{H}}$ indicates a denser environment and thus also a larger amount of material that is involved in the fluorescence emission. This result has been known since the previous studies presented by Torrejón et al. (2010b) and Giménez-García et al. (2015). Compared to these works, we extended the sample of measurements by including Suzaku data, which provide consistent results in a broader range of X-ray luminosity.

The plots in Fig. 1 also confirm the interesting feature mentioned by Giménez-García et al. (2015, see their Fig. 10) that all SFXTs are systematically less absorbed than most of the classical SgXBs, but we show here in addition that the SFXTs are characterized, on average, by $\mathrm{K}_{\alpha}$ iron lines with significantly lower EWs ${ }^{7}$. These two results together indicate that the accretion environment around the compact objects in the SFXTs is systematically less dense than that in classical systems. As mentioned by Giménez-García et al. (2016), this difference can be explained either by assuming that the stellar winds in the SFXTs

\footnotetext{
7 The bulk of the SFXT observations we analyzed are in the quiescent or, at most, in the intermediate state, in which the luminosity is lower than $10^{35} \mathrm{erg} \mathrm{s}^{-1}$.
} 


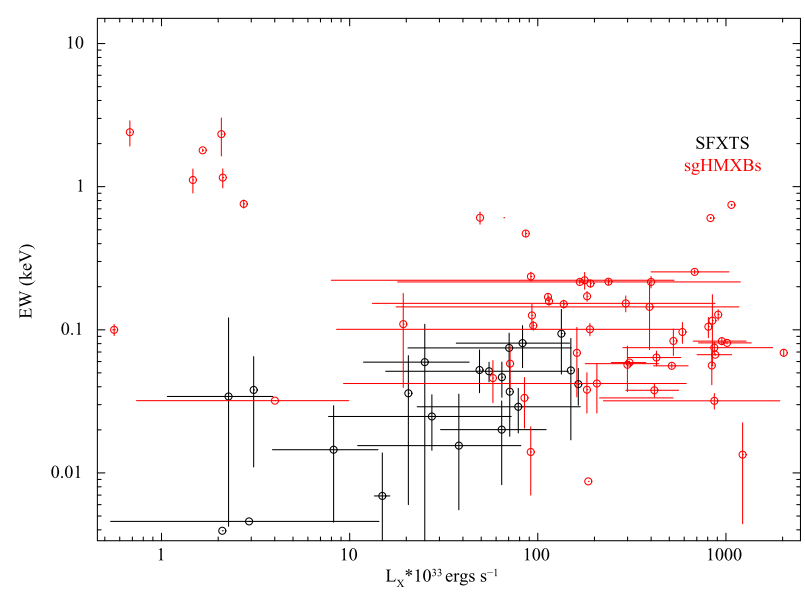

Fig. 2. Equivalent width of the $\mathrm{Fe} \mathrm{K}_{\alpha}$ line vs. the X-ray luminosity for all sources analyzed in this paper. We indicated classical SgXBs in red and SFXTs in black.

are less powerful than those in classical $\mathrm{SgXBs}$, or that the interaction between the compact object and the stellar wind in these two classes of sources is not the same.

A search for systematic differences in the winds of the supergiant companions in SFXTs and in classical SgXBs has been attempted by several authors in the literature, but there is no strong evidence in favor of this hypothesis (Martínez-Núñez et al. 2017). A complication that has so far prevented a detailed study of the stellar winds in these systems is the fact that they are highly absorbed and located at much larger distances with respect to the close-by supergiants for which UV and optical observations provided a great wealth of information on the structure and composition of their winds (see, e.g., Sundqvist et al. 2011, for a recent review). As there does not seem to be an evident dichotomy between spectral classes of supergiants in SFXTs and classical systems, we concentrate in the following paragraphs on the idea that the different interaction between the compact object with the wind of the companion drives the discrepancy between the average absorption column density and iron line EW in these systems.

Detailed studies of classical SgXBs have demonstrated that the irradiation of high energy emission from the compact object can significantly affect the velocity of the surrounding wind, as the latter is radiatively driven and the photoionization by the compact object reduces the main acceleration force of the wind (Ho \& Arons 1987; Manousakis et al. 2012; Krtička et al. 2015; Krtička \& Kubát 2016). In the case of Vela X-1, the prototype of classical $\mathrm{SgXBs}$, a drop of the wind velocity as large as a factor of $\sim 3$ has been inferred from the measured velocity shifts of the emission lines from highly ionized ions close to the NS (using observations performed with X-ray gratings spectrometers; Watanabe et al. 2006).

In the simplistic case of a smooth and symmetrical stellar wind, it is expected that a reduction in the velocity from $v$ to $v^{\prime}=f v$, with $f<1$, leads to an increase in the local density by a factor of $1 / f$ (assuming that the mass loss rate from the supergiant does not change; see, e.g., Sako et al. 2003). These variations can be even larger in the case of structured winds, in which dense clumps transport the bulk of the wind material and could be already endowed with much lower velocities compared to the surrounding inter-clump medium (Oskinova et al. 2012). As the photoionization of the stellar wind steeply increases with the X-ray luminosity and it is particularly effective above $\gtrsim 10^{35}$ $10^{36} \mathrm{erg} \mathrm{s}^{-1}$ (see, e.g., Ducci et al. 2010, and references therein), we suggest that the most likely explanation for the lower density medium around the compact objects in the SFXT is due to the lack of an efficient photoionization of the stellar wind compared to classical systems. This is in line with the widely agreed scenario that accretion in the SFXTs is inhibited for most of the time either by centrifugal or magnetic barrier or by an inefficient settling accretion regime. Their X-ray emission is thus only sporadically achieving the required intensity to substantially slow down the stellar wind and increase the density around the compact object. We note that this would contribute to reducing the X-ray luminosity of the SFXTs even further because the cross section of the compact object for the capture of the stellar wind is inversely proportional to the square of the wind velocity and a smaller cross section implies a reduced mass accretion rate (Frank et al. 2002). In classical SgXBs, the mechanisms inhibiting accretion are unlikely to be at work for a substantial amount of time, and thus we expect these systems to have larger X-ray luminosities and slower winds close to the compact object.

An important assumption in the considerations above is that the bulk of the fluorescence emission leading to the measurable iron $\mathrm{K}_{\alpha}$ lines in both the SFXTs and SgXBs is provided by material around the compact object rather than from the rest of the stellar wind surrounding the binary. This assumption is supported by the rapid variability of the iron line EW measured in several of these sources, as commented in the correspondingly published works (see the cases of, e.g., OAO 1657-415, IGR J17544-2619, and IGR J18410-0535; Pradhan et al. 2014; Rampy et al. 2009; Bozzo et al. 2011).

Our plot of the iron line EW versus the X-ray luminosity for both classical SgXBs and SFXT (Fig. 2) does not confirm the anticorrelation previously reported by Torrejón et al. (2010b) and Giménez-García et al. (2015). We argue that this is most likely due to the larger number of observations and broader range of the X-ray luminosity exploited in the present work thanks to the addition of all available $S u z a k u$ data.

Acknowledgements. The data for this work has been obtained through the High Energy Astrophysics Science Archive (HEASARC) Online Service provided by NASA/GSFC. We would also like to thank the anonymous referee for his/her invaluable comments and suggestions.

\section{References}

Anders, E., \& Grevesse, N. 1989, Geochim. Cosmochim. Acta, 53, 197 Bamba, A., Yokogawa, J., Ueno, M., Koyama, K., \& Yamauchi, S. 2001, PASJ, 53, 1179

Bhalerao, V., Romano, P., Tomsick, J., et al. 2015, MNRAS, 447, 2274

Bozzo, E., Falanga, M., \& Stella, L. 2008, ApJ, 683, 1031

Bozzo, E., Giunta, A., Cusumano, G., et al. 2011, A\&A, 531, A130

Bozzo, E., Bhalerao, V., Pradhan, P., et al. 2016, A\&A, 596, A16

Bozzo, E., Bernardini, F., Ferrigno, C., et al. 2017, A\&A, 608, A128

Chakrabarty, D., Wang, Z., Juett, A. M., Lee, J. C., \& Roche, P. 2002, ApJ, 573, 789

Chaty, S., \& Rahoui, F. 2012, ApJ, 751, 150

Chaty, S., Rahoui, F., Foellmi, C., et al. 2008, A\&A, 484, 783

Coley, J. B., Corbet, R. H. D., \& Krimm, H. A. 2015, ApJ, 808, 140

D'Aì, A., La Parola, V., Cusumano, G., et al. 2011, A\&A, 529, A30

Drave, S. P., Bird, A. J., Townsend, L. J., et al. 2012, A\&A, 539, A21

Drave, S. P., Bird, A. J., Sidoli, L., et al. 2014, MNRAS, 439, 2175

Ducci, L., Sidoli, L., \& Paizis, A. 2010, MNRAS, 408, 1540

Ducci, L., Doroshenko, V., Sasaki, M., et al. 2013, A\&A, 559, A135

Filliatre, P., \& Chaty, S. 2004, ApJ, 616, 469

Frank, J., King, A., \& Raine, D. J. 2002, Accretion Power in Astrophysics, 3rd edn. (Cambridge: Cambridge University Press), 398

George, I. M., \& Fabian, A. C. 1991, MNRAS, 249, 352

Giménez-García, A., Torrejón, J. M., Eikmann, W., et al. 2015, A\&A, 576, A108

Giménez-García, A., Shenar, T., Torrejón, J. M., et al. 2016, A\&A, 591, A26 
P. Pradhan et al.: SFXTs versus classical SgXBs: Does the difference lie in the companion wind?

Grebenev, S. 2008, Proc. 7th INTEGRAL Workshop, 83

Ho, C., \& Arons, J. 1987, ApJ, 316, 283

Inoue, H. 1985, Space Sci. Rev., 40, 317

in't Zand, J. J. M. 2005, A\&A, 441, L1

Islam, N., \& Paul, B. 2014, MNRAS, 441, 2539

Jansen, F., Lumb, D., Altieri, B., et al. 2001, A\&A, 365, L1

Kaper, L., Lamers, H. J. G. L. M., Ruymaekers, E., van den Heuvel, E. P. J., \& Zuiderwijk, E. J. 1995, A\&A, 300, 446

Koyama, K., Tsunemi, H., Dotani, T., et al. 2007, PASJ, 59, 23

Krtička, J., \& Kubát, J. 2016, Adv. Space Res., 58, 710

Krtička, J., Kubát, J., \& Krtičková, I. 2015, A\&A, 579, A111

Malacaria, C., Mihara, T., Santangelo, A., et al. 2016, A\&A, 588, A100

Manousakis, A., Walter, R., \& Blondin, J. M. 2012, A\&A, 547, A20

Martínez-Núñez, S., Torrejón, J. M., Kühnel, M., et al. 2014, A\&A, 563, A70

Martínez-Núñez, S., Kretschmar, P., Bozzo, E., et al. 2017, Space Sci. Rev., submitted

Megier, A., Strobel, A., Galazutdinov, G. A., \& Krełowski, J. 2009, A\&A, 507, 833

Mitsuda, K., Bautz, M., Inoue, H., et al. 2007, PASJ, 59, 1

Morel, T., \& Grosdidier, Y. 2005, MNRAS, 356, 665

Nespoli, E., Fabregat, J., \& Mennickent, R. E. 2008, A\&A, 486, 911

Oskinova, L. M., Feldmeier, A., \& Kretschmar, P. 2012, MNRAS, 421, 2820

Pellizza, L. J., Chaty, S., \& Negueruela, I. 2006, A\&A, 455, 653

Pradhan, P., Maitra, C., Paul, B., Islam, N., \& Paul, B. C. 2014, MNRAS, 442, 2691

Rahoui, F., Chaty, S., Lagage, P.-O., \& Pantin, E. 2008, A\&A, 484, 801
Rampy, R. A., Smith, D. M., \& Negueruela, I. 2009, ApJ, 707, 243

Reig, P., Chakrabarty, D., Coe, M. J., et al. 1996, A\&A, 311, 879

Reig, P., Negueruela, I., Papamastorakis, G., Manousakis, A., \& Kougentakis, T. 2005, A\&A, 440, 637

Reynolds, A. P., Bell, S. A., \& Hilditch, R. W. 1992, MNRAS, 256, 631

Romano, P., Bozzo, E., Mangano, V., et al. 2015, A\&A, 576, L4

Sadakane, K., Hirata, R., Jugaku, J., et al. 1985, ApJ, 288, 284

Sako, M., Kahn, S. M., Paerels, F., et al. 2003, ArXiv e-prints [arXiv: astro-ph/0309503]

Sguera, V., Hill, A. B., Bird, A. J., et al. 2007, A\&A, 467, 249

Sguera, V., Drave, S. P., Sidoli, L., et al. 2013, A\&A, 556, A27

Shakura, N., Postnov, K., Kochetkova, A., \& Hjalmarsdotter, L. 2012, MNRAS, 420, 216

Suchy, S., Fürst, F., Pottschmidt, K., et al. 2012, ApJ, 745, 124

Sundqvist, J. O., Puls, J., Feldmeier, A., \& Owocki, S. P. 2011, A\&A, 528, A64

Tomsick, J. A., Chaty, S., Rodriguez, J., et al. 2006, ApJ, 647, 1309

Torrejón, J. M., Negueruela, I., Smith, D. M., \& Harrison, T. E. 2010a, A\&A 510, A61

Torrejón, J. M., Schulz, N. S., Nowak, M. A., \& Kallman, T. R. 2010b, ApJ, 715, 947

Verner, D. A., Ferland, G. J., Korista, K. T., \& Yakovlev, D. G. 1996, ApJ, 465, 487

Walter, R., \& Zurita Heras, J. 2007, A\&A, 476, 335

Walter, R., Lutovinov, A. A., Bozzo, E., \& Tsygankov, S. S. 2015, A\&ARv, 23, 2

Watanabe, S., Sako, M., Ishida, M., et al. 2006, ApJ, 651, 421

Yamauchi, S., Aoki, T., Hayashida, K., et al. 1995, PASJ, 47, 189 


\section{Appendix A: Spectral fits results}

In this section, we report the details of the spectral fits performed on all observations of classical SgXBs and SFXTs used in this paper. We provide all the absorption and power-law parameters used for fits in Table A.1, while the individual spectra are shown in Figs. A.1-A.5 (together with the best-fit models and the residuals from the fit). All uncertainties are provided at $90 \%$ confidence limit.

Table A.1. Absorption and power-law parameters used for spectral fits of classical SgXBs (S. No 1-55) and SFXTs (S. No 56-76) used in this paper.

\begin{tabular}{|c|c|c|c|c|c|c|c|c|c|c|c|}
\hline No & Source & OBSID (Mission) & $\begin{array}{c}N_{\mathrm{H} 1} \\
10^{22} \text { atoms cm } \\
\end{array}$ & $\begin{array}{c}N_{\mathrm{H} 2} \\
10^{22} \text { atoms cm}{ }^{-2}\end{array}$ & $C_{V}$ & $\Gamma$ & $\begin{array}{c}\text { Emission Line } \\
(\mathrm{keV})\end{array}$ & $\begin{array}{c}\text { EW } \\
(\mathrm{keV})\end{array}$ & $\chi_{\text {red }}^{2} / \mathrm{dof}$ & $\begin{array}{c}\text { Flux (1-10 keV) } \\
10^{-11} \mathrm{ergs} \mathrm{cm}^{-2} \mathrm{~s}^{-1}\end{array}$ & $\begin{array}{c}\text { Distance } \\
(\mathrm{kpc})\end{array}$ \\
\hline \multicolumn{12}{|c|}{ Classical SgXBs } \\
\hline (1) & IGR J00370+6122 & 0501450101 (XMM) & $0.81 \pm 0.03$ & $3.20 \pm 0.28$ & $0.62 \pm 0.02$ & $1.60 \pm 0.03$ & $6.42 \pm 0.10$ & $0.014 \pm 0.007$ & $1.48 / 169$ & $7.48 \pm 0.05$ & 3.3 (Reig et al. 2005) \\
\hline (2) & 4U 0114+65 & 406017010 (Suzaku) & $2.99 \pm 0.15$ & $8.00 \pm 0.80$ & $0.64 \pm 0.03$ & $1.05 \pm 0.03$ & $\begin{array}{c}6.437 \pm 0.014 \\
7.08 \pm 0.04\end{array}$ & $\begin{array}{c}0.0319 \pm 0.004 \\
0.020 \pm 0.005\end{array}$ & $1.01 / 211$ & $14.44 \pm 0.06$ & $7.0 \pm 3.6$ (Reig et al. 1996) \\
\hline (3) & Vela $X-1^{*}$ & 0406430201 (XMM) & $3.29 \pm 0.25$ & $22.43 \pm 2.55$ & $0.31 \pm 0.02$ & $1.00 \pm 0.01$ & $\begin{array}{c}6.419 \pm 0.005 \\
6.71 \pm 0.02 \\
7.05 \pm 0.01\end{array}$ & $\begin{array}{l}0.067 \pm 0.001 \\
0.007 \pm 0.002 \\
0.019 \pm 0.001\end{array}$ & $1.29 / 101$ & $214.99 \pm 0.91$ & $1.9 \pm 0.2$ (Sadakane et al. 1985) \\
\hline (4) & & 0111030101 (XMM) & $8.96 \pm 1.00$ & $13.37 \pm 1.30$ & 0.95 & $1.04 \pm 0.02$ & $6.361 \pm 0.004$ & $0.059 \pm 0.003$ & $1.45 / 90$ & $75.23 \pm 0.15$ & $1.9 \pm 0.2$ (Sadakane et al. 1985$)$ \\
\hline (5) & & 403045010 (Suzaku) & $2.63 \pm 0.11$ & - & - & $1.22 \pm 0.01$ & $\begin{array}{c}6.394 \pm 0.003 \\
7.07 \pm 0.01\end{array}$ & $\begin{array}{l}0.056 \pm 0.002 \\
0.019 \pm 0.001\end{array}$ & $1.42 / 159$ & $126.29 \pm 0.39$ & $1.9 \pm 0.2$ (Sadakane et al. 1985) \\
\hline (6) & GX 301-2* & $0555200301^{1}$ (XMM) & $30.39 \pm 0.09$ & $70.93 \pm 0.40$ & $0.84 \pm 0.01$ & $0.65 \pm 0.01$ & $\begin{array}{c}6.40 \pm 0.01 \\
7.06 \pm 0.002\end{array}$ & $\begin{array}{l}0.604 \pm 0.006 \\
0.129 \pm 0.002\end{array}$ & $1.53 / 103$ & $79.2 \pm 1.33$ & 3.04 (Kaper et al. 1995) \\
\hline (7) & & $0555200401^{2}$ (XMM) & $39.11 \pm 0.11$ & $72.14 \pm 0.95$ & $0.82 \pm 0.02$ & $0.74 \pm 0.01$ & $\begin{array}{l}6.42 \pm 0.02 \\
7.09 \pm 0.01\end{array}$ & $\begin{array}{l}0.746 \pm 0.009 \\
0.181 \pm 0.003\end{array}$ & $1.51 / 1366$ & $102.30 \pm 0.50$ & 3.04 (Kaper et al. 1995) \\
\hline (8) & & $403044020^{3}($ Suzaku $)$ & $22.49 \pm 2.22$ & $18.45 \pm-3.00$ & $0.61 \pm 0.17$ & $0.99 \pm 0.02$ & $\begin{array}{c}6.39 \pm \\
7.09 \pm 0.02\end{array}$ & $\begin{array}{c}0.127 \pm \\
0.017 \pm 0.003\end{array}$ & $1.35 / 155$ & $87.044 \pm 0.25$ & 3.04 (Kaper et al. 1995) \\
\hline (9) & & 403044010 (Suzaku) & $14.95 \pm 1.40$ & $52.76 \pm 3.48$ & 0.95 & $1.03 \pm 0.11$ & $6.38 \pm 0.01$ & $0.219 \pm 0.014$ & $0.92 / 160$ & $16.31 \pm 0.71$ & 3.04 (Kaper et al. 1995) \\
\hline (10) & $4 \mathrm{U} 1538-522^{e}$ & 0152780201 (XMM) & $0.64 \pm 0.06$ & $20.05 \pm 2.05$ & $0.74 \pm 0.04$ & $0.64 \pm 0.09$ & $\begin{array}{c}6.446 \pm 0.021 \\
6.92 \pm 0.01\end{array}$ & $\begin{array}{c}0.6067 \pm 0.060 \\
0.069 \pm 0.021\end{array}$ & $1.39 / 148$ & $1.06 \pm 0.01$ & $6.4 \pm 1$ (Reynolds et al. 1992) \\
\hline (11) & & 407068010 (Suzaku) & $1.48 \pm 0.02$ & - & - & $1.19 \pm 0.01$ & $6.423 \pm 0.009$ & $0.069 \pm 0.005$ & $0.98 / 215$ & $43.79 \pm 0.16$ & $6.4 \pm 1$ (Reynolds et al. 1992) \\
\hline (12) & IGR J16207-5129 & 0402920201 (XMM) & $3.03 \pm 1.14$ & $6.38 \pm 1.10$ & $0.83 \pm 0.15$ & $1.11 \pm 0.09$ & $6.43 \pm 0.04$ & $0.033 \pm 0.012$ & $1.28 / 166$ & $2.09 \pm 0.02$ & $6.10_{-3.50}^{+8.90}$ (Nespoli et al. 2008) \\
\hline (13) & & 402065020 (Suzaku) & $11.30 \pm 0.89$ & - & - & $1.23 \pm 0.11$ & $6.427 \pm 0.035$ & $0.058 \pm 0.019$ & $1.17 / 116$ & $1.75 \pm 0.03$ & $6.10_{-3.50}^{+8.90}$ (Nespoli et al. 2008) \\
\hline (14) & IGR J16318-4848 & 0154750401 (XMM) & $153.28 \pm 18.50$ & - & - & $0.34 \pm 0.44$ & $\begin{array}{c}6.389 \pm 0.003 \\
7.24 \pm 0.06\end{array}$ & $\begin{array}{c}2.33 \pm 0.92 \\
0.623 \pm 0.360\end{array}$ & $1.33 / 59$ & $0.725 \pm 0.001$ & 2.60 (Filliatre \& Chaty 2004) \\
\hline (15) & & 0201000201 (XMM) & $120.63 \pm 11.00$ & - & - & $0.45 \pm 0.27$ & $\begin{array}{c}6.403 \pm 0.004 \\
7.05 \pm 0.02\end{array}$ & $\begin{array}{c}1.17 \pm 0.18 \\
0.258 \pm 0.052\end{array}$ & $1.36 / 66$ & $0.732 \pm 0.012$ & 2.60 (Filliatre \& Chaty 2004) \\
\hline (16) & & 0201000301 (XMM) & $133.30 \pm 27.50$ & - & - & $0.35 \pm 0.64$ & $\begin{array}{l}6.41 \pm 0.01 \\
7.05 \pm 0.01\end{array}$ & $\begin{array}{c}2.55 \pm 1.94 \\
0.258 \pm 0.156\end{array}$ & $0.97 / 101$ & $0.235 \pm 0.002$ & 2.60 (Filliatre \& Chaty 2004) \\
\hline (17) & & $401094010^{4}$ (Suzaku) & $124.16 \pm 9.05$ & - & - & $1.07 \pm 0.20$ & $\begin{array}{c}6.395 \pm 0.005 \\
7.09 \pm 0.04\end{array}$ & $\begin{array}{l}0.758 \pm 0.050 \\
0.108 \pm 0.008\end{array}$ & $1.64 / 124$ & $0.922 \pm 0.008$ & 2.60 (Filliatre \& Chaty 2004) \\
\hline (18) & & $0201000401^{5}$ (XMM) & $114.56 \pm 8.50$ & - & - & $0.71 \pm 0.33$ & $\begin{array}{c}6.404 \pm 0.001 \\
7.05 \pm 0.03\end{array}$ & $\begin{array}{c}1.115 \pm 0.213 \\
0.298 \pm 0.055\end{array}$ & $1.12 / 60$ & $0.507 \pm 0.007$ & 2.60 (Filliatre \& Chaty 2004) \\
\hline (19) & & $0742270201^{6}$ (XMM) & $146.16 \pm 4.50$ & - & - & $0.66 \pm 0.02$ & $\begin{array}{c}6.439 \pm 0.003 \\
7.11 \pm 0.02\end{array}$ & $\begin{array}{c}1.76 \pm 0.04 \\
0.432 \pm 0.077\end{array}$ & $1.26 / 101$ & $0.570 \pm 0.047$ & 2.60 (Filliatre \& Chaty 2004) \\
\hline (20) & IGR J16320-4751 & 0128531101 (XMM) & $20.36 \pm 2.45$ & - & - & $1.09 \pm 0.20$ & $6.43 \pm 0.15$ & $0.109 \pm 0.070$ & $0.89 / 105$ & $1.392 \pm 0.031$ & 3.5 (Rahoui et al. 2008) \\
\hline (21) & & 0556140101 (XMM) & $26.19 \pm 1.15$ & - & - & $0.41 \pm 0.08$ & $\begin{array}{c}6.420 \pm 0.002 \\
7.04 \pm 0.03\end{array}$ & $\begin{array}{l}0.243 \pm 0.017 \\
0.049 \pm 0.011\end{array}$ & $1.30 / 99$ & $6.68 \pm 0.08$ & 3.5 (Rahoui et al. 2008) \\
\hline (22) & & 0556140201 (XMM) & $18.88 \pm 0.68$ & - & - & $0.309 \pm 0.056$ & $\begin{array}{c}6.420 \pm 0.004 \\
7.01 \pm 0.06\end{array}$ & $\begin{array}{l}0.210 \pm 0.012 \\
0.061 \pm 0.022\end{array}$ & $1.21 / 123$ & $13.75 \pm 0.20$ & 3.5 (Rahoui et al. 2008) \\
\hline (23) & & 0556140301 (XMM) & $18.68 \pm 0.65$ & - & - & $0.34 \pm 0.06$ & $\begin{array}{l}6.42 \pm 0.01 \\
7.01 \pm 0.06\end{array}$ & $\begin{array}{c}0.176 \pm 0.014 \\
0.071\end{array}$ & 1.18/119 & $0.132 \pm 0.001$ & 3.5 (Rahoui et al. 2008) \\
\hline (24) & & 0556140401 (XMM) & $22.30 \pm 0.95$ & - & - & 0.56 & $\begin{array}{l}6.41 \pm 0.01 \\
7.23 \pm 0.30\end{array}$ & $\begin{array}{l}0.157 \pm 0.001 \\
0.108 \pm 0.112\end{array}$ & $1.37 / 117$ & $8.29 \pm 0.07$ & 3.5 (Rahoui et al. 2008) \\
\hline (25) & & 0556140501 (XMM) & $21.92 \pm 1.80$ & - & - & $0.56 \pm 0.14$ & $6.41 \pm 0.02$ & $0.125 \pm 0.025$ & $1.07 / 89$ & $6.71 \pm 0.13$ & 3.5 (Rahoui et al. 2008) \\
\hline (26) & & 0556140601 (XMM) & $19.13 \pm 0.28$ & - & - & $0.18 \pm 0.03$ & $\begin{array}{c}6.420 \pm 0.004 \\
6.99 \pm 0.03\end{array}$ & $\begin{array}{l}0.216 \pm 0.010 \\
0.117 \pm 0.012\end{array}$ & $1.52 / 122$ & $0.172 \pm 0.001$ & 3.5 (Rahoui et al. 2008) \\
\hline (27) & & 0556140701 (XMM) & $41.95 \pm 2.90$ & - & - & $0.022 \pm 0.131$ & $\begin{array}{c}6.420 \pm 0.005 \\
7.03 \pm 0.05\end{array}$ & $\begin{array}{l}0.469 \pm 0.023 \\
0.167 \pm 0.042\end{array}$ & $1.37 / 96$ & $6.23 \pm 0.05$ & 3.5 (Rahoui et al. 2008) \\
\hline (28) & & 0556140801 (XMM) & $19.86 \pm 1.02$ & - & - & $0.54 \pm 0.09$ & $\begin{array}{c}6.409 \pm 0.004 \\
7.07 \pm 0.24\end{array}$ & $\begin{array}{c}0.170 \pm 0.010 \\
0.298 \pm 0.202\end{array}$ & $1.16 / 113$ & $8.17 \pm 0.07$ & 3.5 (Rahoui et al. 2008) \\
\hline (29) & & 0556141001 (XMM) & $18.27 \pm 0.94$ & - & - & $0.45 \pm 0.08$ & $\begin{array}{c}6.408 \pm 0.010 \\
7.07 \pm 0.17\end{array}$ & $\begin{array}{l}0.151 \pm 0.010 \\
0.286 \pm 0.118\end{array}$ & $0.95 / 113$ & $9.91 \pm 0.20$ & 3.5 (Rahoui et al. 2008) \\
\hline (30) & & 0201700301 (XMM) & $14.41 \pm 0.48$ & - & - & $0.49 \pm 0.05$ & $\begin{array}{c}6.397 \pm 0.001 \\
7.01 \pm 0.20\end{array}$ & $\begin{array}{l}0.107 \pm 0.007 \\
0.365 \pm 0.308\end{array}$ & $1.50 / 125$ & $6.83 \pm 0.02$ & 3.5 (Rahoui et al. 2008) \\
\hline $\begin{array}{l}(31) \\
\text { (32) } \\
\text { (33) }\end{array}$ & IGR J16393-4643 & $\begin{array}{l}0206380201 \text { (XMM) } \\
0604520201 \text { (XMM) } \\
404056010 \text { (Suzaku) }\end{array}$ & $\begin{array}{l}25.51 \pm 1.28 \\
25.28 \pm 2.95 \\
29.36 \pm 1.75\end{array}$ & $\begin{array}{l}\overline{-} \\
\overline{-}\end{array}$ & $\begin{array}{l}- \\
\overline{-}\end{array}$ & $\begin{array}{l}0.61 \pm 0.09 \\
0.65 \pm 0.21 \\
1.12 \pm 0.13\end{array}$ & $\begin{array}{c}6.393 \pm 0.027 \\
6.48 \pm 0.06 \\
6.390 \pm 0.048\end{array}$ & $\begin{array}{l}0.084 \pm 0.017 \\
0.069 \pm 0.035 \\
0.057 \pm 0.020\end{array}$ & $\begin{array}{l}1.05 / 107 \\
0.71 / 94 \\
1.16 / 129\end{array}$ & $\begin{array}{l}4.14 \pm 0.04 \\
1.27 \pm 0.02 \\
2.33 \pm 0.03\end{array}$ & $\begin{array}{l}10.6 \text { (Chaty et al. 2008) } \\
10.6 \text { (Chaty et al. 2008) } \\
10.6 \text { (Chaty et al. 2008) }\end{array}$ \\
\hline $\begin{array}{l}(34) \\
\text { (35) }\end{array}$ & IGR J16418-4532 & $\begin{array}{l}0405180501 \text { (XMM) } \\
0679810201 \text { (XMM) }\end{array}$ & $\begin{array}{l}3.51 \pm 0.89 \\
1.42 \pm 0.14\end{array}$ & $\begin{array}{l}5.76 \pm 0.99 \\
3.93 \pm 0.31\end{array}$ & $\begin{array}{c}0.64 \pm 0.17 \\
0.95\end{array}$ & $\begin{array}{l}1.47 \pm 0.11 \\
0.99 \pm 0.04\end{array}$ & $\begin{array}{c}6.4 \\
6.39 \pm 0.20\end{array}$ & $\begin{array}{c}0.008 \\
0.013 \pm 0.009\end{array}$ & $\begin{array}{l}0.95 / 133 \\
1.19 / 154\end{array}$ & $\begin{array}{l}0.97 \pm 0.02 \\
6.41 \pm 0.08\end{array}$ & $\begin{array}{l}13 \text { (Rahoui et al. 2008) } \\
13 \text { (Rahoui et al. 2008) }\end{array}$ \\
\hline (36) & IGR J16493-4348 & 401054010 (Suzaku) & $8.26 \pm 1.85$ & - & - & $1.41 \pm 0.21$ & $6.452 \pm 0.093$ & $0.046 \pm 0.001$ & $1.61 / 67$ & $1.42 \pm 0.03$ & 6 (Martínez-Núñez et al. 2017) \\
\hline (37) & OAO $1657-415$ & 406011010 (Suzaku) & $17.28 \pm 1.35$ & $37.14 \pm 6.57$ & $0.57 \pm 0.07$ & $0.87 \pm 0.04$ & $\begin{array}{c}6.459 \pm 0.002 \\
7.14 \pm 0.04\end{array}$ & $\begin{array}{l}0.254 \pm 0.008 \\
0.098 \pm 0.007\end{array}$ & $1.31 / 162$ & $14.72 \pm 0.15$ & 1.5 (Chakrabarty et al. 2002) \\
\hline (38) & $4 \mathrm{U} 1700-37$ & 0083280101 (XMM) & $3.88 \pm 0.19$ & $5.89 \pm 0.44$ & $0.67 \pm 0.04$ & $1.09 \pm 0.03$ & $\begin{array}{c}6.43 \pm 0.01 \\
7.10\end{array}$ & $\begin{array}{c}0.037 \pm 0.004 \\
0.021\end{array}$ & $1.59 / 154$ & $81.97 \pm 0.54$ & $2.12 \pm 0.34$ (Megier et al. 2009) \\
\hline (39) & & $0083280201^{7}$ (XMM) & $1.36 \pm 0.08$ & $5.55 \pm 1.20$ & $0.27 \pm 0.04$ & $0.75 \pm 0.03$ & $\begin{array}{c}6.43 \pm \\
7.11 \pm 0.03\end{array}$ & $\begin{array}{l}0.083 \pm 0.004 \\
0.014 \pm 0.003\end{array}$ & $1.42 / 137$ & $187.09 \pm 1.39$ & $2.12 \pm 0.34$ (Megier et al. 2009) \\
\hline (40) & & 0083280301 (XMM) & $6.10 \pm 0.25$ & $10.74 \pm 0.46$ & 0.95 & $1.11 \pm 0.03$ & $\begin{array}{l}6.43 \pm 0.01 \\
7.16 \pm 0.06\end{array}$ & $\begin{array}{l}0.064 \pm 0.007 \\
0.013 \pm 0.005\end{array}$ & $1.38 / 157$ & $84.41 \pm 0.92$ & $2.12 \pm 0.34$ (Megier et al. 2009) \\
\hline (41) & & $401058010^{8}$ (Suzaku) & $1.91 \pm 0.10$ & $3.92 \pm 0.25$ & $0.61 \pm 0.03$ & $0.23 \pm 0.09$ & $\begin{array}{l}6.41 \pm 0.01 \\
7.07 \pm 0.02\end{array}$ & $\begin{array}{l}0.079 \pm 0.001 \\
0.018 \pm 0.002\end{array}$ & $1.42 / 214$ & $197.03 \pm 0.32$ & $2.12 \pm 0.34$ (Megier et al. 2009) \\
\hline
\end{tabular}

Notes. ${ }^{(1)}$ Additional emission lines centroid energy (uncertainty), EW (uncertainty) = $3.72(0.01), 0.029(0.001), 5.41(0.02), 0.013(0.002), 6.29$ (0.02), 0.033 (0.001), $7.47(0.01), 0.065(0.002), 8.11(0.04), 0.11(0.01) .{ }^{(2)}$ Additional emission lines centroid energy (uncertainty), EW (uncertainty) $=3.73(0.01), 0.091(0.004), 5.43(0.01), 0.015(0.002), 6.20(0.01), 0.015(0.001), 7.50(0.01), 0.015(0.001), 8.23(0.02), 0.23(0.01) .{ }^{(3)}$ Additional emission lines centroid energy (uncertainty), EW (uncertainty) $=7.46(0.02), 0.016(0.002) .{ }^{(4)}$ Additional emission line centroid energy (uncertainty), EW (uncertainty) $=7.39(0.11), 0.138(0.065) .{ }^{(5)}$ Additional emission line centroid energy (uncertainty), EW (uncertainty) $=7.47$ (0.06), 0.145 (0.083). ${ }^{(6)}$ Additional emission line centroid energy (uncertainty), EW (uncertainty) $=7.52(0.02), 0.287$ (0.047). ${ }^{(7)}$ bbKT (uncertainty), bbnorm (uncertainty) $=0.10(0.02), 0.0053(0.0021){ }^{(8)}$ Ecut (uncertainty) $=7.57(1.00) .{ }^{(*)}$ The X-ray spectra of Vela X-1 and GX $301-2$ are dominated below $3 \mathrm{keV}$ by orbital-dependent emission lines and soft excesses which origin is still largely debated (see Martínez-Núñez et al. 2014; Suchy et al. 2012; Islam \& Paul 2014 and references therein). As the interpretation of these features is beyond the scope of the current paper, we excluded the energy range 1-3 keV from the fit of the spectra of these two sources. We verified that this has no quantitative impact on the reported results and on their average absorption column density measured. ${ }^{e}$ Eclipse times are excluded. 
P. Pradhan et al.: SFXTs versus classical SgXBs: Does the difference lie in the companion wind?

Table A.1. continued.

\begin{tabular}{|c|c|c|c|c|c|c|c|c|c|c|c|}
\hline No & Source & OBSID (Mission) & $\begin{array}{c}N_{\mathrm{H} 1} \\
10^{22} \text { atoms cm } \\
\end{array}$ & $\begin{array}{c}N_{\mathrm{H} 2} \\
10^{22} \text { atoms cm } \\
\end{array}$ & $C_{V}$ & $\bar{\Gamma}$ & $\begin{array}{c}\begin{array}{c}\text { Emission Line } \\
(\mathrm{keV})\end{array} \\
\end{array}$ & $\begin{array}{l}\begin{array}{l}\mathrm{EW} \\
(\mathrm{keV})\end{array} \\
\end{array}$ & $\overline{\chi_{\text {red }}^{2} / \mathrm{dof}}$ & $\begin{array}{c}\text { Flux }(1-10 \mathrm{keV}) \\
10^{-11} \mathrm{ergs} \mathrm{cm}^{-2} \mathrm{~s}^{-1}\end{array}$ & $\begin{array}{c}\text { Distance } \\
(\mathrm{kpc})\end{array}$ \\
\hline (42) & EXO 1722-363 & 0405640301 (XMM) & $11.77 \pm 0.45$ & - & - & $\begin{array}{r}\text { SFXTs } \\
0.82 \pm 0.08\end{array}$ & $6.41 \pm 0.01$ & $\begin{array}{l}0.216 \pm 0.025 \\
0.049+0.019\end{array}$ & $1.07 / 115$ & $5.19 \pm 0.12$ & 6-10.5 (Martínez-Núñez et al. 2017) \\
\hline (43) & & 0405640401 (XMM) & $26.96 \pm 2.15$ & - & - & $0.64 \pm 0.15$ & $\begin{array}{l}6.41 \pm 0.01 \\
7.14 \pm 0.12\end{array}$ & $\begin{array}{l}0.221 \pm 0.033 \\
0.057 \pm 0.001\end{array}$ & $1.04 / 84$ & $2.31 \pm 0.19$ & 6-10.5 (Martínez-Núñez et al. 2017) \\
\hline (44) & & 0405640701 (XMM) & $28.06 \pm 1.20$ & - & - & $0.71 \pm 0.09$ & $\begin{array}{l}6.45 \pm 0.02 \\
7.13 \pm 0.02\end{array}$ & $\begin{array}{l}0.100 \pm 0.010 \\
0.052 \pm 0.017\end{array}$ & $1.01 / 132$ & $2.46 \pm 0.06$ & 6-10.5 (Martínez-Núñez et al. 2017) \\
\hline (45) & & 0405640801 (XMM) & $13.83 \pm 4.28$ & $19.85 \pm 4.41$ & $0.83 \pm 0.17$ & $1.01 \pm 0.12$ & $\begin{array}{l}6.42 \pm 0.01 \\
7.09 \pm 0.02\end{array}$ & $\begin{array}{l}0.153 \pm 0.019 \\
0.069 \pm 0.034\end{array}$ & $0.95 / 116$ & $3.81 \pm 0.22$ & 6-10.5 (Martínez-Núñez et al. 2017) \\
\hline (46) & & 0405640901 (XMM) & $19.95 \pm 0.98$ & - & - & $0.93 \pm 0.09$ & $\begin{array}{l}6.42 \pm 0.05 \\
7.09 \pm 0.35\end{array}$ & $\begin{array}{c}0.046 \pm 0.016 \\
0.033\end{array}$ & $0.99 / 112$ & $2.68 \pm 0.10$ & 6-10.5 (Martínez-Núñez et al. 2017) \\
\hline (47) & & 0206380401 (XMM) & $15.63 \pm 3.50$ & - & - & $0.42 \pm 0.30$ & $\begin{array}{l}6.40 \pm 0.05 \\
7.07\end{array}$ & $\begin{array}{l}0.144 \pm 0.072 \\
0.058\end{array}$ & $0.97 / 54$ & $5.10 \pm 0.95$ & 6-10.5 (Martínez-Núñez et al. 2017) \\
\hline (48) & SAX J1802.7-2017 & 0206380601 (XMM) & $7.41 \pm 0.44$ & & & $0.78 \pm 0.08$ & $6.50 \pm 0.14$ & $0.116 \pm 0.060$ & $1.21 / 125$ & $4.87 \pm 0.05$ & 12.4 (Martínez-Núñez et al. 2017) \\
\hline (49) & & 0745060401 (XMM) & $4.65 \pm 1.11$ & $14.64 \pm 4.50$ & $0.71 \pm 0.09$ & $0.78 \pm 0.11$ & 6.38 & $0.038 \pm 0.012$ & $1.38 / 146$ & $1.05 \pm 0.01$ & 12.4 (Martínez-Núñez et al. 2017) \\
\hline (50) & & 0745060501 (XMM) & $2.42 \pm 0.27$ & $7.44 \pm 2.11$ & $0.56 \pm 0.07$ & $1.07 \pm 0.09$ & 6.39 & $0.096 \pm 0.016$ & $1.03 / 146$ & $3.38 \pm 0.03$ & 12.4 (Martínez-Núñez et al. 2017) \\
\hline (51) & & 0745060601 (XMM) & $2.21 \pm 0.85$ & $6.49 \pm 0.60$ & $0.89 \pm 0.08$ & $0.67 \pm 0.06$ & 6.39 & $0.105 \pm 0.017$ & $1.07 / 149$ & $4.64 \pm 0.03$ & 12.4 (Martínez-Núñez et al. 2017) \\
\hline (52) & & 0745060801 (XMM) & $1.75 \pm 0.05$ & - & - & $0.98 \pm 0.02$ & $6.41 \pm 0.04$ & $0.057 \pm 0.015$ & $1.02 / 152$ & $4.83 \pm 0.04$ & 12.4 (Martínez-Núñez et al. 2017) \\
\hline (53) & XTE J1855-026 & 409022010 (Suzaku) & $4.54 \pm 1.75$ & $14.75 \pm 3.50$ & $0.63 \pm 0.18$ & $1.36 \pm 0.08$ & $\begin{array}{c}6.408 \pm 0.011 \\
7.15 \pm 0.08\end{array}$ & $\begin{array}{l}0.100 \pm 0.009 \\
0.023 \pm 0.009\end{array}$ & $1.27 / 215$ & $6.74 \pm 0.11$ & $8.6 \pm 0.8$ (Coley et al. 2015) \\
\hline (54) & $4 \mathrm{U} 1909+07$ & 405073010 (Suzaku) & $5.54 \pm 0.60$ & $6.87 \pm 2.15$ & $0.49 \pm 0.15$ & $1.33 \pm 0.05$ & $\begin{array}{c}6.424 \pm 0.012 \\
7.10\end{array}$ & $\begin{array}{l}0.075 \pm 0.007 \\
0.018 \pm 0.005\end{array}$ & $1.37 / 109$ & $15.64 \pm 0.26$ & $7.00 \pm 3.0($ Morel \& Grosdidier 2005) \\
\hline $\begin{array}{l}(55) \\
(56)\end{array}$ & $\begin{array}{l}\text { IGR J19140+0951 } \\
\text { IGR J11215-5952 }\end{array}$ & $\begin{array}{l}0761690301(\mathrm{XMM}) \\
0405181901(\mathrm{XMM})\end{array}$ & $\begin{array}{l}7.67 \pm 0.46 \\
0.66 \pm 0.04\end{array}$ & $\begin{array}{c}- \\
2.27 \pm 0.49\end{array}$ & $\stackrel{-}{-}$ & $\begin{array}{l}1.79 \pm 0.11 \\
1.19 \pm 0.05\end{array}$ & $\begin{array}{c}6.4 \\
6.46 \pm 0.06\end{array}$ & $\begin{array}{c}0.032 \\
0.042 \pm 0.012\end{array}$ & $\begin{array}{l}0.95 / 116 \\
1.16 / 163\end{array}$ & $\begin{array}{c}0.29 \pm 0.004 \\
3.77 \pm 0.04\end{array}$ & $\begin{array}{l}\text { 2-5 (Martínez-Núñez et al. 2017) } \\
\text { 6.2 (Martínez-Núñez et al. 2017) }\end{array}$ \\
\hline (57) & IGR J16195-4945 & $401056010($ Suzaku $)$ & $8.63 \pm 0.83$ & - & - & $1.19 \pm 0.11$ & $6.38 \pm 0.06$ & $0.052 \pm 0.019$ & $1.10 / 171$ & $1.74 \pm 0.08$ & 5 (Tomsick et al. 2006) \\
\hline $\begin{array}{l}(58) \\
(59) \\
(60)\end{array}$ & IGR J16328-4726 & $\begin{array}{l}0728560201 \text { (XMM) } \\
0728560301 \text { (XMM) } \\
0654190201 \text { (XMM) }\end{array}$ & $\begin{array}{c}4.23 \pm 0.55 \\
12.86 \pm 0.63 \\
16.88 \pm 1.10\end{array}$ & $\begin{array}{c}12.06 \pm 0.88 \\
- \\
-\end{array}$ & $\begin{array}{l}0.97 \\
- \\
-\end{array}$ & $\begin{array}{l}1.36 \pm 0.06 \\
1.40 \pm 0.09 \\
1.45 \pm 0.12\end{array}$ & $\begin{array}{c}6.41 \pm 0.05 \\
6.42 \pm 0.07 \\
6.4\end{array}$ & $\begin{array}{l}0.029 \pm 0.011 \\
0.075 \pm 0.020 \\
0.015 \pm 0.010\end{array}$ & $\begin{array}{l}1.14 / 133 \\
0.98 / 118 \\
1.09 / 106\end{array}$ & $\begin{array}{l}1.65 \pm 0.02 \\
1.47 \pm 0.03 \\
0.79 \pm 0.04\end{array}$ & $\begin{array}{l}\text { 3-10 (Martínez-Núñez et al. 2017) } \\
\text { 3-10 (Martínez-Núñez et al. 2017) } \\
\text { 3-10 (Martínez-Núnez et al. 2017) }\end{array}$ \\
\hline (61) & IGR J16479-4514e & $406078010($ Suzaku $)$ & $2.33 \pm 1.11$ & $6.06 \pm 2.16$ & $0.90 \pm 0.09$ & $1.44 \pm 0.14$ & 6.34 & $0.081 \pm 0.026$ & $0.73 / 47$ & $1.30 \pm 0.03$ & (Chaty et al. 2008) \\
\hline $\begin{array}{l}(62) \\
(63)\end{array}$ & IGR J17354-3255 & $\begin{array}{l}0701230101 \text { (XMM) } \\
0701230701 \text { (XMM) }\end{array}$ & $\begin{array}{l}2.17 \pm 0.53 \\
5.22 \pm 0.21\end{array}$ & $\begin{array}{c}5.73 \pm 1.12 \\
-\end{array}$ & $\begin{array}{c}0.96 \\
-\end{array}$ & $\begin{array}{l}1.33 \pm 0.09 \\
1.20 \pm 0.05\end{array}$ & $\begin{array}{c}6.41 \pm 0.04 \\
6.407 \pm 0.065\end{array}$ & $\begin{array}{l}0.036 \pm 0.019 \\
0.094 \pm 0.045\end{array}$ & $\begin{array}{l}0.94 / 145 \\
1.10 / 138\end{array}$ & $\begin{array}{l}9.80 \pm 0.53 \\
1.86 \pm 0.43\end{array}$ & $\begin{array}{l}8 \text { (D’Aì et al. 2011) } \\
8 \text { (D'Aì et al. 2011) }\end{array}$ \\
\hline $\begin{array}{l}(64) \\
(65)\end{array}$ & XTE 1739-302 & $\begin{array}{l}0554720101 \text { (XMM) } \\
0561580101 \text { (XMM) }\end{array}$ & $\begin{array}{l}2.08 \pm 0.44 \\
3.28 \pm 0.55\end{array}$ & $\begin{array}{l}3.87 \pm 1.30 \\
7.03 \pm 3.35\end{array}$ & $\begin{array}{l}0.67 \pm 0.16 \\
0.52 \pm 0.15\end{array}$ & $\begin{array}{l}1.96 \pm 0.15 \\
1.95 \pm 0.18\end{array}$ & $\begin{array}{c}6.4 \\
6.42 \pm 0.11\end{array}$ & $\begin{array}{c}0.004 \\
0.038 \pm 0.027\end{array}$ & $\begin{array}{l}0.89 / 122 \\
0.89 / 117\end{array}$ & $\begin{array}{l}0.25 \pm 0.01 \\
0.37 \pm 0.04\end{array}$ & $\begin{array}{l}2.7 \text { (Rahoui et al. 2008) } \\
2.7 \text { (Rahoui et al. 2008) }\end{array}$ \\
\hline $\begin{array}{l}(66) \\
(67) \\
(68) \\
(69)\end{array}$ & IGR J17544-2619 & $\begin{array}{l}0744600101 \text { (XMM) } \\
0679810401 \text { (XMM) } \\
402061010 \text { (Suzaku) } \\
0154750601 \text { (XMM) }\end{array}$ & $\begin{array}{l}0.36 \pm 0.08 \\
1.59 \pm 0.57 \\
1.24 \pm 0.21 \\
1.31 \pm 0.45\end{array}$ & $\begin{array}{l}1.23 \pm 0.13 \\
3.70 \pm 1.71 \\
1.92 \pm 0.30 \\
3.76 \pm 1.21\end{array}$ & $\begin{array}{c}0.95 \\
0.76 \pm 0.15 \\
0.71 \pm 0.11 \\
0.78 \pm 0.10\end{array}$ & $\begin{array}{l}1.19 \pm 0.04 \\
2.60 \pm 0.31 \\
1.26 \pm 0.03 \\
2.31 \pm 0.27\end{array}$ & $\begin{array}{c}6.4 \\
6.40 \\
6.32 \pm 0.08 \\
6.40\end{array}$ & $\begin{array}{c}0.013 \\
0.034 \pm 0.029 \\
0.019 \pm 0.011 \\
0.06 \pm 0.05\end{array}$ & $\begin{array}{c}1.21 / 168 \\
0.79 / 70 \\
0.99 / 150 \\
0.92 / 126\end{array}$ & $\begin{array}{l}0.71 \pm 0.01 \\
0.19 \pm 0.03 \\
5.55 \pm 0.52 \\
2.17 \pm 0.43\end{array}$ & $\begin{array}{l}3.20 \pm 1.00 \text { (Pellizza et al. 2006) } \\
3.20 \pm 1.00 \text { (Pellizza et al. 2006) } \\
3.20 \pm 1.00 \text { (Pellizza et al. 2006) } \\
3.20 \pm 1.00 \text { (Pellizza et al. 2006) }\end{array}$ \\
\hline (70) & SAX J1818.6-1703 & 0693900101 (XMM) & $27.93 \pm 1.15$ & - & - & $0.49 \pm 0.07$ & 6.4 & $0.0069 \pm$ & $1.16 / 139$ & $2.99 \pm 0.06$ & $2.1 \pm 0.1$ (Torrejón et al. 2010a) \\
\hline $\begin{array}{l}(71) \\
(72)\end{array}$ & IGR J18410-0535 & $\begin{array}{l}\text { 0604820301(XMM) } \\
505090010(\text { Suzaku) }\end{array}$ & $\begin{array}{l}2.87 \pm 0.18 \\
1.91 \pm 0.28\end{array}$ & $\begin{aligned} 10.81 & \pm 0.55 \\
5.44 & \pm 1.21\end{aligned}$ & $\begin{array}{c}0.95 \\
0.69 \pm 0.07\end{array}$ & $\begin{array}{l}1.13 \pm 0.05 \\
1.70 \pm 0.07\end{array}$ & $\begin{array}{l}6.44 \pm 0.05 \\
6.40 \pm 0.02\end{array}$ & $\begin{array}{l}0.026 \pm 0.010 \\
0.051 \pm 0.008\end{array}$ & $\begin{array}{l}1.34 / 154 \\
0.96 / 169\end{array}$ & $\begin{array}{l}2.36 \pm 0.02 \\
4.75 \pm 0.12\end{array}$ & $\begin{array}{l}3.2_{-1.5}^{+2.0} \text { (Nespoli et al. 2008) } \\
3.2_{-1.5}^{+2.0} \text { (Nespoli et al. 2008) }\end{array}$ \\
\hline $\begin{array}{l}\text { (73) } \\
\text { (74) }\end{array}$ & IGR J18450-0435 & $\begin{array}{c}0728370801 \text { (Suzaku) } \\
0306170401 \text { (XMM) }\end{array}$ & $\begin{array}{l}2.78 \pm 0.76 \\
1.79 \pm 0.18\end{array}$ & $\begin{array}{l}6.41 \pm 1.15 \\
4.39 \pm 1.20\end{array}$ & $\begin{array}{l}0.84 \pm 0.10 \\
0.55 \pm 0.07\end{array}$ & $\begin{array}{l}1.39 \pm 0.11 \\
1.33 \pm 0.07\end{array}$ & $\begin{array}{l}6.49 \pm 0.09 \\
6.41 \pm 0.03\end{array}$ & $\begin{array}{l}0.036 \pm 0.003 \\
0.048 \pm 0.003\end{array}$ & $\begin{array}{l}1.19 / 132 \\
0.85 / 148\end{array}$ & $\begin{array}{l}1.13 \pm 0.04 \\
3.54 \pm 0.14\end{array}$ & $\begin{array}{l}4 \text { (Yamauchi et al. 1995) } \\
4 \text { (Yamauchi et al. 1995) }\end{array}$ \\
\hline (75) & IGR J18462-0223 & 0651680301 (XMM) & $16.91 \pm 1.20$ & - & - & $1.15 \pm 0.14$ & $6.34 \pm 0.06$ & $0.052 \pm 0.035$ & $0.98 / 97$ & $1.09 \pm 0.02$ & 11 (Sguera et al. 2013) \\
\hline (76) & IGR J18483-0311 & 0694070101 (XMM) & $4.45 \pm 0.75$ & $9.09 \pm 3.65$ & $0.62 \pm 0.12$ & $2.04 \pm 0.17$ & 6.40 & 0.045 & $1.05 / 128$ & $0.32 \pm 0.03$ & 3-4 (Rahoui et al. 2008) \\
\hline
\end{tabular}


A\&A 610, A50 (2018)
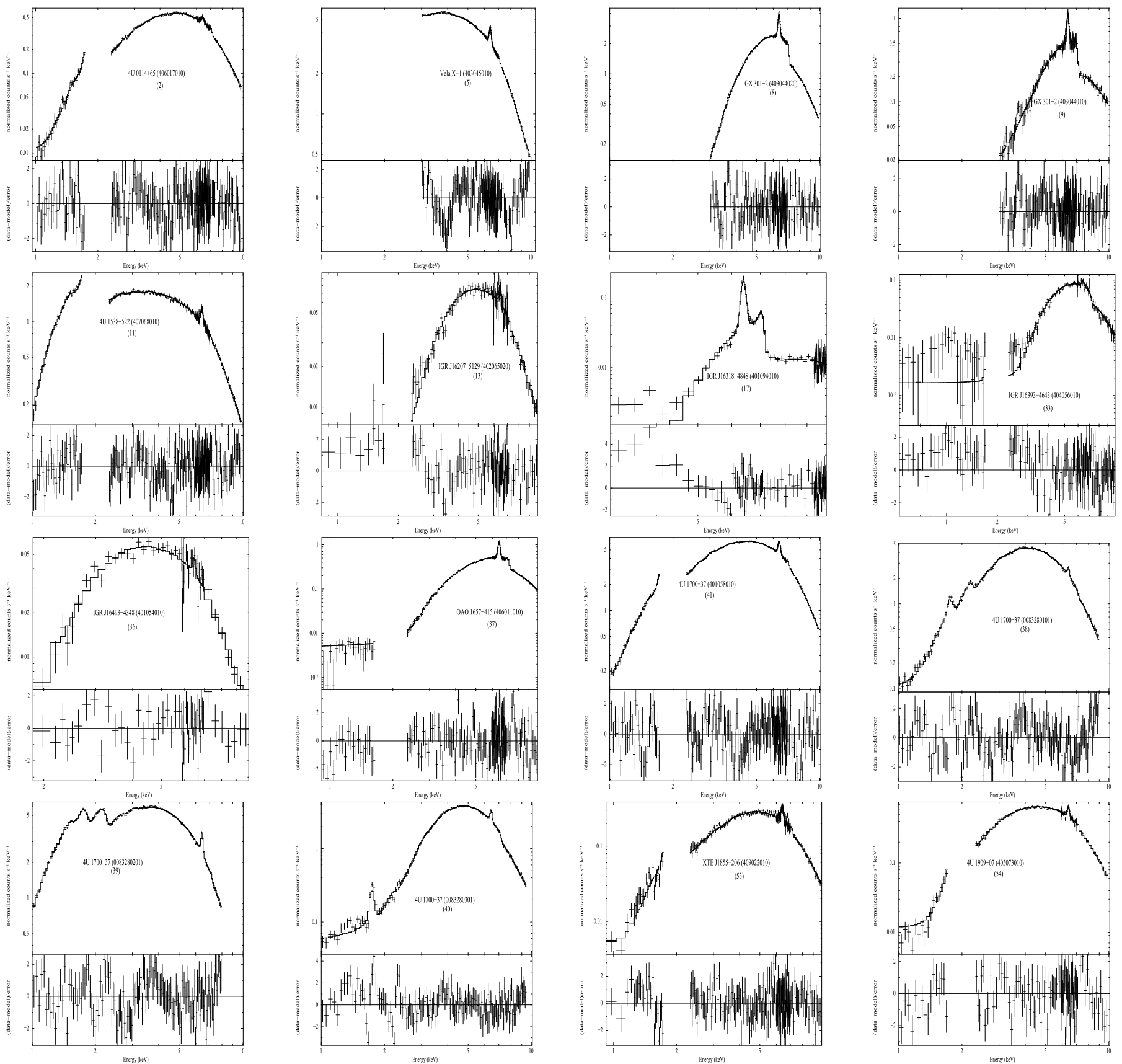

Fig. A.1. Suzaku spectra of all considered classical SgXBs. The source name and the observation ID is indicated in each figure. 
P. Pradhan et al.: SFXTs versus classical SgXBs: Does the difference lie in the companion wind?
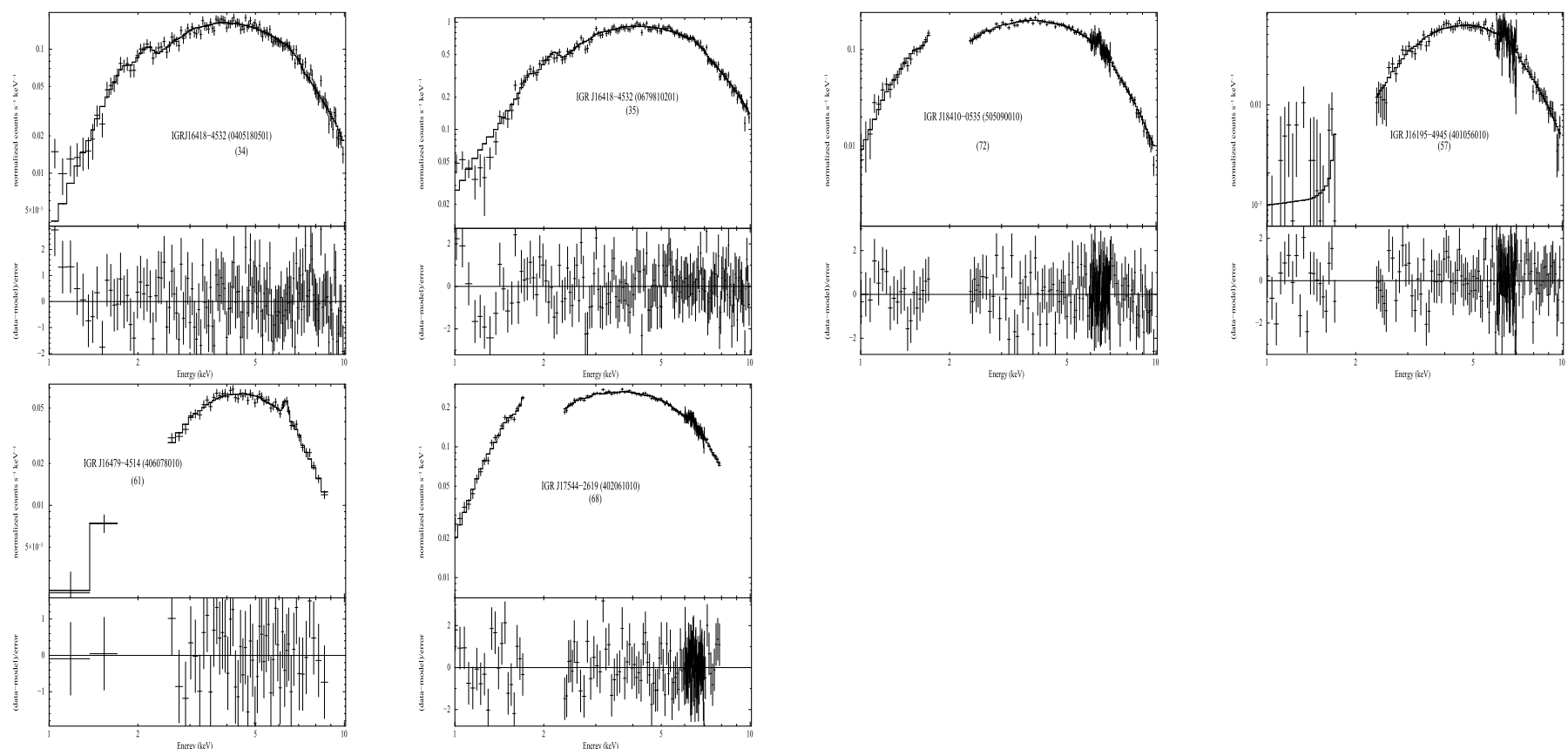

Fig. A.2. Suzaku spectra of all considered SFXTs. The source name and the observation ID is indicated in each figure. 

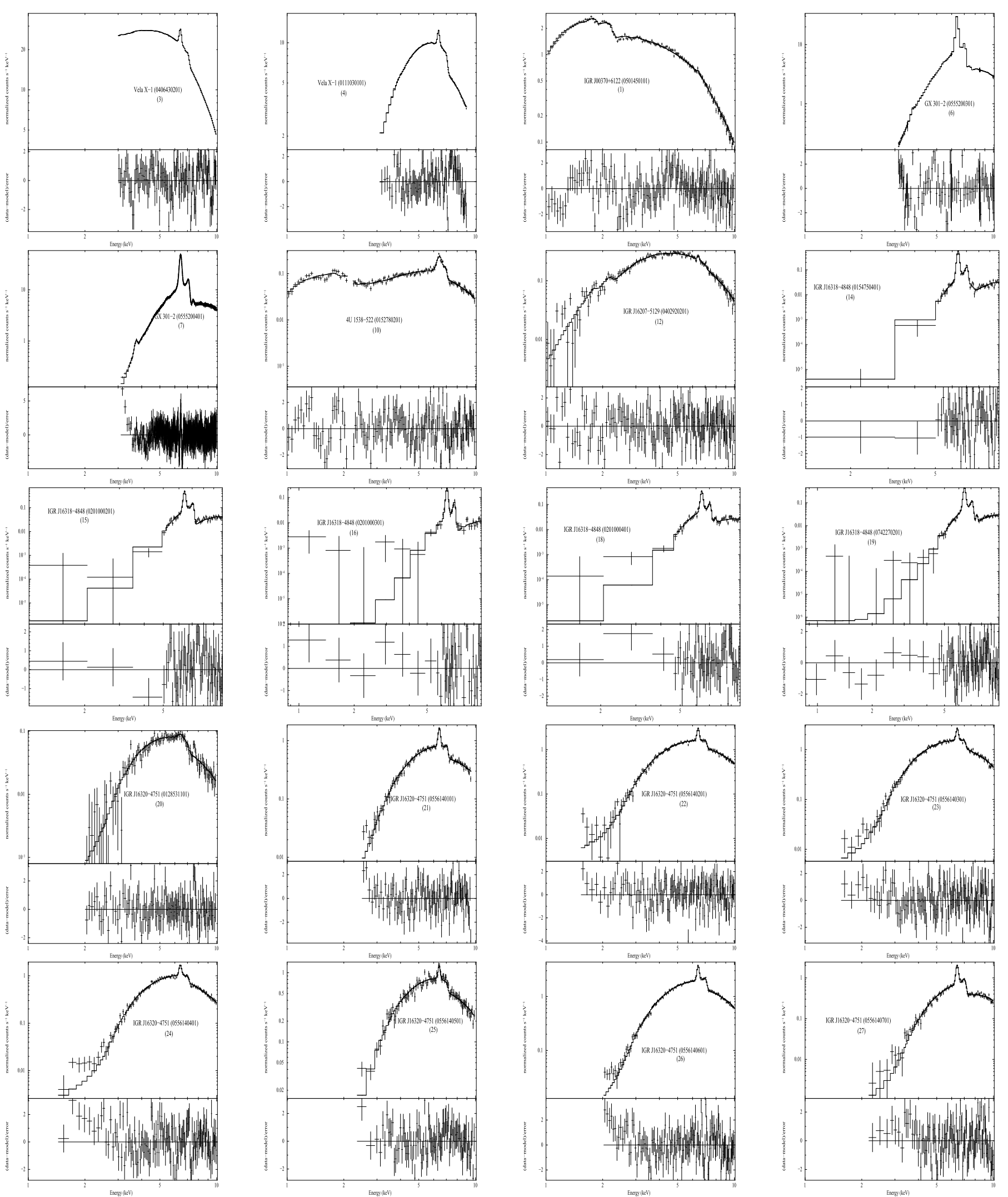

Fig. A.3. XMM-Newton spectra of all considered classical SgXBs. The source name and the observation ID is indicated in each figure. 
P. Pradhan et al.: SFXTs versus classical SgXBs: Does the difference lie in the companion wind?
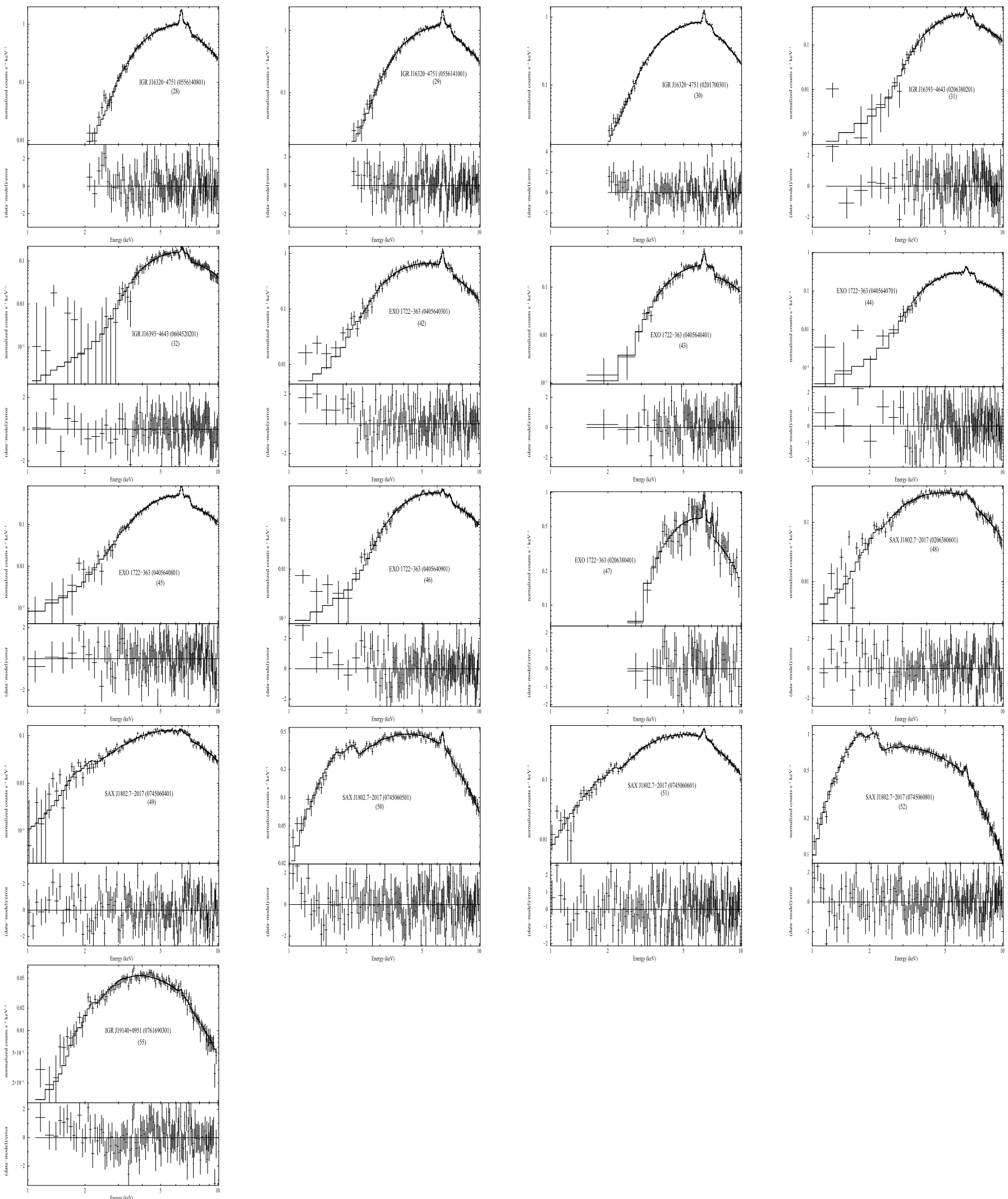

Fig. A.4. XMM-Newton spectra of all considered classical SgXBs. The source name and the observation ID is indicated in each figure. 

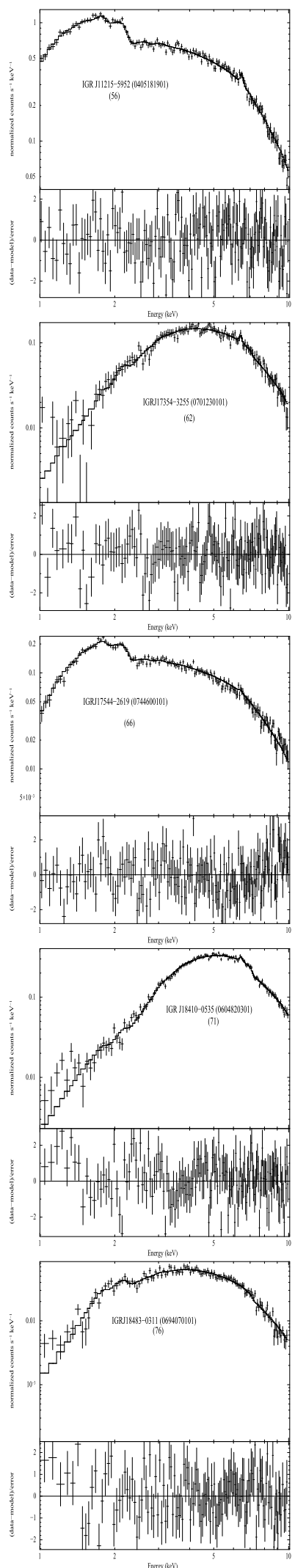
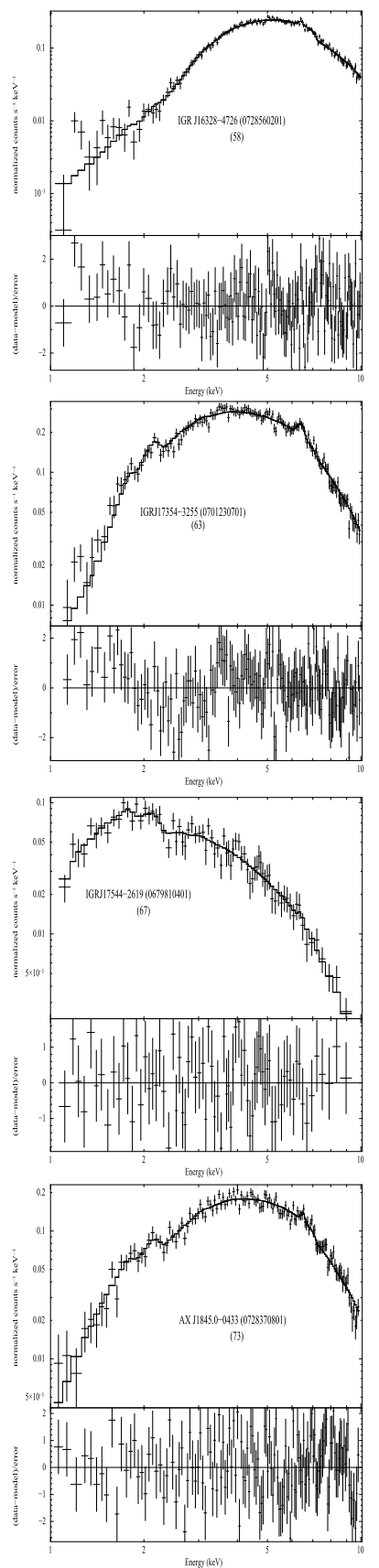
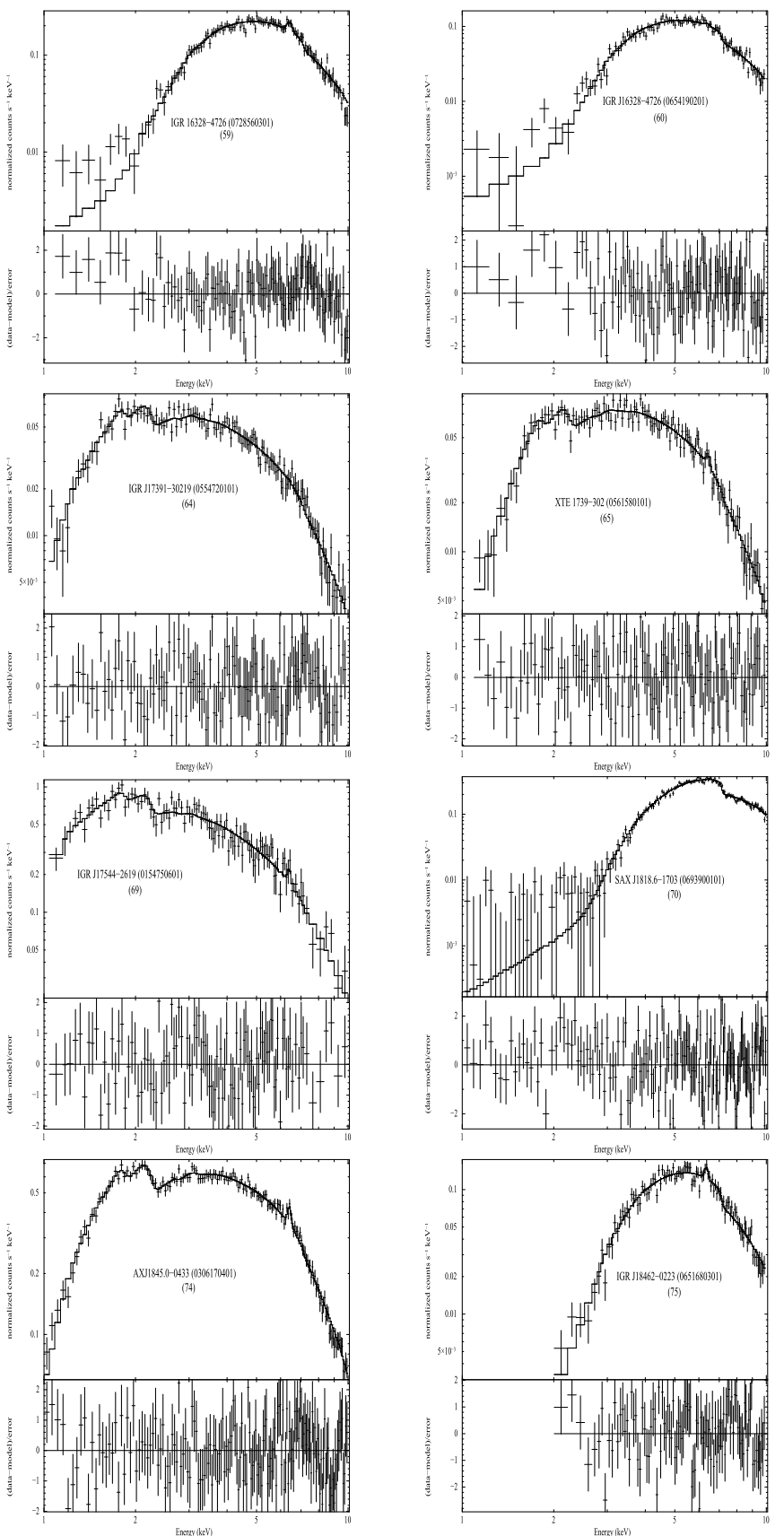

Fig. A.5. XMM-Newton spectra of all considered SFXTs. The source name and the observation ID is indicated in each figure. 